\title{
REGULARITY OF THE SOLUTION TO A NONSTANDARD SYSTEM OF PHASE FIELD EQUATIONS
}

\author{
PIERLUIGI COLLI (*), GIANNI GILARDI $\left(^{*}\right)$, \\ JÜRGEN SPREKELS (**) \\ Nota presentata dal s.c. Gianni Gilardi \\ (Adunanza dell'11 aprile 2013)
}

\begin{abstract}
SunTO. Questa nota riguarda un sistema non standard di equazioni differenziali che descrive la segregazione di fase di due specie. Il sistema nasce in modo naturale nell'analisi asintotica, elaborata di recente da Colli, Gilardi, Krejčí e Sprekels, al tendere a zero del coefficiente di diffusione nell'equazione che governa l'evoluzione del parametro d'ordine. In particolare viene fornito un risultato di buona positura per il sistema limite. Questa nota tratta lo stesso problema limite in un quadro meno generale ma ancora decisamente significativo e fornisce una dimostrazione molto semplice della regolarità della soluzione. Come sottoprodotto viene data una dimostrazione altrettanto semplice dell'unicità.

$$
* * *
$$
\end{abstract}

AвSTRACT. A nonstandard system of differential equations describing two-species phase segregation is considered. This system naturally arises in the asymptotic analysis recently done by Colli, Gilardi, Krejčí, and Sprekels as the diffusion coefficient in the equation governing the evolution of the order parameter tends to zero. In particular, a wellposedness result is proved for the limit system. This paper deals with the above limit problem in a less general but still very significant framework and provides a very simple proof of further regularity for the solution. As a byproduct, a simple uniqueness proof is given as well.

$\left(^{*}\right)$ Dipartimento di Matematica "F. Casorati", Università di Pavia, via Ferrata 1, 27100 Pavia, Italy. E-mail: pierluigi.colli@unipv.it; gianni.gilardi@unipv.it

$\left.{ }^{(* *}\right)$ Weierstraß-Institut für Angewandte Analysis und Stochastik, Mohrenstraße 39, 10117 Berlin, Germany. E-mail: sprekels@wias-berlin.de 


\section{INTRODUCTION}

In this paper, we consider the system

$$
\begin{aligned}
& (1+2 g(\rho)) \partial_{t} \mu+\mu g^{\prime}(\rho) \partial_{t} \rho-\Delta \mu=0 \\
& \partial_{t} \rho+f^{\prime}(\rho)=\mu g^{\prime}(\rho) \\
& \left.\partial_{\nu} \mu\right|_{\Gamma}=0 \\
& \mu(0)=\mu_{0} \quad \text { and } \quad \rho(0)=\rho_{0}
\end{aligned}
$$

in the unknown fields $\mu$ and $\rho$, where the equations (1)-(2) are meant to hold in a bounded domain $\Omega \subset \mathbb{R}^{3}$ with a smooth boundary $\Gamma$ and in some time interval $(0, T)$, and where $\partial_{\nu}$ in (3) denotes the outward normal derivative. In the recent papers $[1,2]$, the well-posedness of the problem (1)-(4) was investigated, and in particular the existence of the solution was proved by considering the system of partial differential equations obtained by replacing the ordinary differential equation (2) by the partial differential equation

$$
\begin{aligned}
& \partial_{t} \rho-\sigma \Delta \rho+f^{\prime}(\rho)=\mu g^{\prime}(\rho) \\
& \text { with the boundary condition }\left.\partial_{\nu} \rho\right|_{\Gamma}=0
\end{aligned}
$$

and performing the asymptotic analysis as $\sigma$ tends to zero. This modified system originates from the model introduced in [3], which describes the phase segregation of two species (atoms and vacancies, say) on a lattice in presence of diffusion and looks like a modification of the well-known Cahn-Hilliard equations (see, e.g., $[4,5]$ ). The state variables are the order parameter $\rho$ (volume density of one of the two species), which of course must attain values in the domain of $f^{\prime}$, and the chemical potential $\mu$, which is required to be nonnegative for physical reasons. This system has been studied in a series of papers and a number of results has been obtained in several directions $[6,7,8,9,10,11]$. Moreover, some of these results hold for a more general system involving a nonlinear elliptic operator in divergence form in equation (1), in place of the Laplacian 
(see $[1,2,12,13])$. In all of the quoted papers, the function $f$ represents a double-well potential. A thermodynamically relevant example is the so-called logarithmic potential defined (up to an additive constant) by the formula

$$
f(\rho)=c_{1}(\rho \log \rho+(1-\rho) \log (1-\rho))+c_{2} \rho(1-\rho) \quad \text { for } \rho \in(0,1),
$$

where $c_{1}$ and $c_{2}$ are positive constant with $c_{2}>2 c_{1}$ in order that $f$ actually presents a double well. However, the class of the admissible potentials could be much wider and includes both the standard doublewell potential defined by

$$
f(\rho)=\frac{1}{4}\left(\rho^{2}-1\right)^{2} \quad \text { for } \rho \in \mathbb{R}
$$

and potentials whose convex part is just proper and lower semicontinuous, and thus possibly non-differentiable, in its effective domain. In the latter case, the monotone part of $f^{\prime}$ is replaced by a multivalued subdifferential and (5) has to be read as a differential inclusion. In [1], such a wide class of potentials is considered, so that the existence result for system (1)-(4) obtained there is very general. However, the solution constructed in this way may be irregular, in principle. Nevertheless, it is unique and a little more regular than expected, at the price that the corresponding proofs are rather complicated.

The present paper deals just with potentials that see example (6) as a prototype, but it provides simple proofs of further regularity. As an application, we give an easy uniqueness proof.

Our paper is organized as follows. In the next section, we list our assumptions and state problem (1)-(4) in a precise form. In the last section, we present and prove our results.

\section{AsSUMPTIONS AND NOTATIONS}

We first introduce precise assumptions on the data for the mathematical problem under investigation. We assume $\Omega$ to be a bounded connected 
open set in $\mathbb{R}^{3}$ with smooth boundary $\Gamma$ (treating lower-dimensional cases would require only minor changes) and let $T \in(0,+\infty)$ stand for a final time. We set

$$
\begin{aligned}
& V:=H^{1}(\Omega), \quad H:=L^{2}(\Omega), \\
& \text { and } \quad W:=\left\{v \in H^{2}(\Omega):\left.\partial_{\nu} v\right|_{\Gamma}=0\right\}
\end{aligned}
$$

and endow the spaces (8) with their standard norms, for which we use a self-explanatory notation like $\|\cdot\|_{V}$. For simplicity, we use the same notation also for powers of these spaces. The symbol $\langle\cdot, \cdot\rangle$ denotes the duality product between $V^{*}$, the dual space of $V$, and $V$ itself. Moreover, for $p \in[1,+\infty]$, we write $\|\cdot\|_{p}$ for the usual norm both in $L^{p}(\Omega)$ and in $L^{p}(Q)$, where $Q:=\Omega \times(0, T)$. For the nonlinearities and the initial data we assume that there exist

$$
\rho_{*}, \rho^{*} \in \mathbb{R} \quad \text { with } \quad \rho_{*}<\rho^{*}
$$

in order that the combined conditions listed below hold.

$$
\begin{aligned}
& f, g:\left[\rho_{*}, \rho^{*}\right] \rightarrow \mathbb{R} \text { are } C^{2} \text { functions } \\
& g(r) \geq 0 \text { and } g^{\prime \prime}(r) \leq 0 \text { for every } r \in\left[\rho_{*}, \rho^{*}\right] \\
& f^{\prime}\left(\rho_{*}\right) \leq 0 \leq g^{\prime}\left(\rho_{*}\right) \text { and } g^{\prime}\left(\rho^{*}\right) \leq 0 \leq f^{\prime}\left(\rho^{*}\right) . \\
& \mu_{0} \in V \cap L^{\infty}(\Omega) \text { and } \mu_{0} \geq 0 \quad \text { a.e. in } \Omega \\
& \rho_{0} \in V \quad \text { and } \rho_{*} \leq \rho_{0} \leq \rho^{*} \quad \text { a.e. in } \Omega
\end{aligned}
$$

Notice that the functions $f, g$, together with their first derivatives, are bounded and Lipschitz continuous. Moreover, we remark that the different assumptions of [1] can be fulfilled by splitting $f$ as $f_{1}+f_{2}$ with $f_{1}$ nonnegative and convex, and suitably extending $f_{1}, f_{2}$, and $g$ to an open interval including $\left[\rho_{*}, \rho^{*}\right]$. In particular, the logarithmic potential (6) fits the above requirements with $\rho_{*}, \rho^{*} \in(0,1)$ and reasonable choices of $g$.

Now, we recall the part that follows from the asymptotic analysis performed in [1] and is of interest for the present paper. 
THEOREM 2.1. Assume that the assumptions (9)-(14) bold. Then there exists at least one pair $(\mu, \rho)$ satisfying

$$
\begin{gathered}
\mu \in L^{\infty}(0, T ; H) \cap L^{2}(0, T ; V) \cap L^{\infty}(Q) \text { and } \mu \geq 0 \text { a.e. in } Q \\
\rho \in H^{1}(0, T ; H) \cap L^{\infty}(0, T ; V) \text { and } \rho_{*} \leq \rho \leq \rho^{*} \text { a.e. in } Q \\
u:=(1+2 g(\rho)) \mu \in W^{1,1}\left(0, T ; V^{*}\right)
\end{gathered}
$$

and solving the problem

$$
\begin{aligned}
& \left\langle\partial_{t} u(t), v\right\rangle+\int_{\Omega} \nabla \mu(t) \cdot \nabla v=\int_{\Omega} \mu(t) g^{\prime}(\rho(t)) \partial_{t} \rho(t) v \\
& \quad \text { for all } v \in V \text { and a.a. } t \in(0, T) \\
& \partial_{t} \rho+f^{\prime}(\rho)=\mu g^{\prime}(\rho) \text { a.e. in } Q \\
& u(0)=\left(1+2 g\left(\rho_{0}\right)\right) \mu_{0} \quad \text { and } \quad \rho(0)=\rho_{0} \quad \text { a.e. in } \Omega \text {. }
\end{aligned}
$$

We observe that the first regularity level for the time derivative of $u$ obtained in [1] is $\partial_{t} u \in L^{4 / 3}\left(0, T ; V^{*}\right)$, that is, a little better than (17). However, one easily sees that $\partial_{t} u \in L^{2}\left(0, T ; V^{*}\right)$ by comparison in (18), on account of (15)-(16) and (10).

Remark 2.2. We can also consider the stronger form of (18),

$$
\begin{aligned}
\int_{\Omega}(1+2 g(\rho(t))) \partial_{t} \mu(t) v & +\int_{\Omega} \mu(t) g^{\prime}(\rho(t)) \partial_{t} \rho(t) v \\
& +\int_{\Omega} \nabla \mu(t) \cdot \nabla v=0
\end{aligned}
$$

for all $v \in V$ and for a.a. $t \in(0, T)$, and observe that it is equivalent to (18) provided that one can apply the Leibniz rule to the time derivative $\partial_{t} u$. This is the case if $\partial_{t} \mu$ exists and belongs to $L^{2}(Q)$. However, Theorem 2.1 does not ensure such a regularity. Moreover, (18) also 
includes the homogenous Neumann boundary condition (3) in a generalized sense.

The aim of this paper is to prove that any solution to problem (18)(20) satisfying the very mild regularity (15)-(17) is in fact much smoother and, in particular, unique.

Now, we list a number of tools and notations used throughout the paper. First of all, we often use the elementary Young inequality,

$$
a b \leq \varepsilon a^{2}+\frac{1}{4 \varepsilon} b^{2} \quad \text { for every } a, b \geq 0 \text { and } \varepsilon>0,
$$

and repeatedly account for the Hölder and Sobolev inequalities. The precise form of the latter we use is the following:

$$
V \subset L^{q}(\Omega) \quad \text { and } \quad\|v\|_{q} \leq C\|v\|_{V} \quad \text { for every } v \in V \text { and } q \in[1,6],
$$

where $C$ depends only on $\Omega$. Moreover, the above embedding is compact if $q<6$, and the compactness inequality

$$
\|v\|_{q} \leq \varepsilon\|\nabla v\|_{2}+C_{q, \varepsilon}\|v\|_{2} \quad \text { for every } v \in V, q \in[1,6) \text {, and } \varepsilon>0,
$$

holds with a constant $C_{q, \varepsilon}$ depending on $\Omega, q$, and $\varepsilon$, only. Furthermore, we exploit the embeddings

$$
\begin{aligned}
L^{\infty}(0, T ; H) \cap L^{2}(0, T ; V) & \subset L^{\infty}(0, T ; H) \cap L^{2}\left(0, T ; L^{6}(\Omega)\right) \\
& \subset L^{10 / 3}(Q),
\end{aligned}
$$

as well as the corresponding inequality

$$
\|v\|_{L^{10 / 3}(Q)} \leq C\left(\|v\|_{L^{\infty}(0, T ; H)}+\|v\|_{L^{2}(0, T ; V)}\right),
$$

which follow from combining the Sobolev embedding $V \subset L^{6}(\Omega)$ and the well-known interpolation inequality for $L^{p}$ spaces. Again, $C$ depends only on $\Omega$. Finally, we set

$$
Q_{t}:=\Omega \times(0, t) \quad \text { for } t \in(0, T]
$$


and use the same symbol small-case $c$ for different constants, that may only depend on $\Omega$, the final time $T$, the nonlinearities $f$ and $g$, and the properties of the data involved in the statements at hand. A notation like $c_{\varepsilon}$ signals a constant that also depends on the parameter $\varepsilon$. The reader should keep in mind that the meaning of $c$ and $c_{\varepsilon}$ may change from line to line and even within the same chain of inequalities, whereas those constants we need to refer to are always denoted by capital letters, just like $C$ in (23) and in (26).

\section{REGULARITY}

In this section, we prove regularity results for the solution to problem (18)-(20) under the assumption that the conditions (9)-(14) hold (we often avoid writing this). In order to help the reader, we sketch our strategy. We fix any solution $(\mu, \rho)$ to problem (18)-(20) satisfying the regularity requirements (15)-(17) and recall that all of the nonlinear terms involving $\rho$ are bounded. Moreover, $\mu$ is bounded, too (cf. (15)). Thus, (19) implies that even $\partial_{t} \rho$ is bounded. Now, we set

$$
a:=1+2 g(\rho) \text { and } b:=\mu g^{\prime}(\rho) \partial_{t} \rho
$$

and notice that $\partial_{t} a=2 g^{\prime}(\rho) \partial_{t} \rho$. Hence, we have

$$
\begin{gathered}
a \in L^{\infty}(0, T ; V) \cap L^{\infty}(Q), \\
\partial_{t} a \in L^{\infty}(Q), \\
b \in L^{\infty}(Q), \\
a \geq 1 \quad \text { a.e. in } Q .
\end{gathered}
$$

Next, we introduce the associated linear problem

$$
\begin{aligned}
& \left\langle\partial_{t}(a z)(t), v\right\rangle+\int_{\Omega} \nabla z(t) \cdot \nabla v \\
& =\int_{\Omega} b(t) v \text { for all } v \in V \text { and a.a. } t \in(0, T)
\end{aligned}
$$




$$
(a z)(0)=\left(1+2 g\left(\rho_{0}\right)\right) \mu_{0},
$$

whose unknown $z$ is required to satisfy

$$
z \in L^{\infty}(0, T ; H) \cap L^{2}(0, T ; V) \text { and } a z \in W^{1,1}\left(0, T ; V^{*}\right),
$$

and observe that $z=\mu$ is a solution. Then, we prove that (30)-(31) has a unique solution $z$ satisfying (32). This implies the following. If we regularize (30)-(31) and perform some a priori estimates on the solution to the regularized problem then these estimates still hold for any weak limit. On the other hand, such a limit must be $\mu$ due to uniqueness. This entails further regularity for $\mu$. Once the regularity obtained for $\mu$ is sufficiently high, we can even prove uniqueness in a simple way. We observe that uniqueness for (30)-(31) is not straightforward. Indeed, (30) is a very weak form (due to the very low regularity (32)) of the homogeneous Neumann boundary value problem for the equation

$$
\partial_{t}(a z)-\Delta z=b,
$$

which is formally uniformly parabolic. However, the equation is not presented in divergence form, and $a$ might be discontinuous since no continuity for $\rho$ is known. At this point, we can start with our program.

LEMMA 3.1. Let $(\mu, \rho)$ be a solution to (18)-(20) satisfying (15)-(17), and let $a$ and $b$ be defined by (28). Then problem (30)-(31) bas a unique solution $z$ satisfying (32), and this solution coincides with $\mu$.

PROOF. Clearly, $\mu$ satisfies (32) and solves (30)-(31). As far as uniqueness is concerned, we can deal with the correponding homogeneous problem, by linearity. Thus, we fix any $z$ satisfying (32) that solves (30)(31), where $b$ is replaced by zero on the right-hand side of (30) and the initial value in (31) is zero. Then, we introduce the adjoint problem of finding $v$ such that 


$$
\begin{aligned}
& v \in H^{1}(0, T ; H) \cap L^{2}(0, T ; W) \subset C^{0}([0, T] ; V) \\
& -a \partial_{t} v-\Delta v=z \quad \text { a.e. in } Q \quad \text { and } \quad v(T)=0 .
\end{aligned}
$$

It is easily seen that (33)-(34) has a (unique) solution since $z \in$ $L^{2}(0, T ; H)$. We use such a solution $v$ as a test function for $z$, observing that the integration by parts formula

$$
\int_{0}^{T}\left\langle\partial_{t} w(t), \phi(t)\right\rangle d t=\langle w(T), \phi(T)\rangle-\langle w(0), \phi(0)\rangle-\int_{0}^{T}\left\langle\partial_{t} \phi(t), w(t)\right\rangle d t
$$

is actually valid if $w \in L^{2}(0, T ; V) \cap W^{1,1}\left(0, T ; V^{*}\right)$ and $\phi \in$ $C^{0}([0, T] ; V) \cap H^{1}\left(0, T ; V^{*}\right)$. Thus, the choice $\phi=v$ is allowed by (33), Hence, we have

$$
\begin{aligned}
0= & \int_{0}^{T}\left\langle\partial_{t}(a z)(t), v(t)\right\rangle d t+\int_{Q} \nabla z \cdot \nabla v \\
= & \langle(a z)(T), v(T)\rangle-\langle(a z)(0), v(0)\rangle-\int_{0}^{T}\left\langle\partial_{t} v(t),(a z)(t)\right\rangle d t \\
& -\int_{Q} z \Delta v \\
= & \int_{Q}\left(-a \partial_{t} v-\Delta v\right) z=\int_{Q} z^{2},
\end{aligned}
$$

which implies that $z=0$.

COROLLARY 3.2. If $z \in H^{1}(0, T ; H) \cap L^{\infty}(0, T ; V) \cap L^{2}(0, T ; W)$ solves

$$
a \partial_{t} z+\partial_{t} a z-\Delta z=b \quad \text { a.e. in } Q \text { and } z(0)=\mu_{0},
$$

then it holds $z=\mu$.

PROOF. Indeed, our assumptions on $z$ and (35) imply both (32) and (30)-(31). Thus, the previous lemma gives the result. 
THEOREM 3.3. Assume that the assumptions (9)-(14) are fulfilled, and let $(\mu, \rho)$ be a solution to (18)-(20) satisfying (15)-(17). Then $\mu$ also satisfies

$$
\mu \in H^{1}(0, T ; H) \cap L^{2}(0, T ; W) .
$$

In particular, $\mu \in C^{0}([0, T] ; V)$.

PROOF. Thanks to (29), there exist two sequences $\left\{a_{n}\right\}$ and $\left\{b_{n}\right\}$ of $C^{1}$ functions such that

$$
\begin{aligned}
& a_{n} \rightarrow a, \quad \partial_{t} a_{n} \rightarrow \partial_{t} a, \quad b_{n} \rightarrow b \quad \text { strongly in } L^{2}(Q) \\
& \left|a_{n}\right|+\left|\partial_{t} a_{n}\right|+\left|b_{n}\right| \leq c \quad \text { and } \quad a_{n} \geq 1 \quad \text { a.e. in } Q
\end{aligned}
$$

Then, we consider for any $n \in \mathbb{N}$ the following regularization of problem (35):

$$
a_{n} \partial_{t} z_{n}+\partial_{t} a_{n} z_{n}-\Delta z_{n}=b_{n} \quad \text { a.e. in } Q \quad \text { and } \quad z_{n}(0)=\mu_{0},
$$

complemented with the homogeneous Neumann boundary condition. Due to the regularity of the coefficients and assumption (13) on $\mu_{0}$, it is easy to see that this problem has a (unique) solution $z_{n}$ satisfying

$$
z_{n} \in H^{1}(0, T ; H) \cap L^{\infty}(0, T ; V) \cap L^{2}(0, T ; W) .
$$

We add $z_{n}$ to both sides of the equation for convenience. Then, we multiply the resulting equality by $\partial_{t} z_{n}$ and integrate over $Q_{t}$ for any $t \in$ $(0, T)$. Owing to $(38)$, we easily obtain that

$$
\begin{aligned}
\int_{Q_{t}}\left|\partial_{t} z_{n}\right|^{2}+\frac{1}{2}\left\|z_{n}(t)\right\|_{V}^{2} & \leq \frac{1}{2} \int_{\Omega}\left|\nabla \mu_{0}\right|^{2}+\int_{Q_{t}}\left(b_{n}+z_{n}-\partial_{t} a_{n} z_{n}\right) \partial_{t} z_{n} \\
& \leq \frac{1}{2} \int_{\Omega}\left|\nabla \mu_{0}\right|^{2}+\frac{1}{2} \int_{Q_{t}}\left|\partial_{t} z_{n}\right|^{2}+c \int_{Q_{t}}\left(1+\left|z_{n}\right|^{2}\right) \\
& \leq c+\frac{1}{2} \int_{Q_{t}}\left|\partial_{t} z_{n}\right|^{2}+c \int_{0}^{t}\left\|z_{n}(s)\right\|_{V}^{2} d s .
\end{aligned}
$$


By rearranging and applying the Gronwall lemma, we immediately conclude that

$$
\left\|\partial_{t} z_{n}\right\|_{L^{2}(0, T ; H)}+\left\|z_{n}\right\|_{L^{\infty}(0, T ; V)} \leq c .
$$

Moreover, by comparison in (39), we also find an estimate for $\left\|\Delta z_{n}\right\|_{H}$. So, the above bounds and standard regularity results for elliptic equations yield the estimate

$$
\left\|z_{n}\right\|_{H^{1}(0, T ; H)}+\left\|z_{n}\right\|_{L^{\infty}(0, T ; V)}+\left\|z_{n}\right\|_{L^{2}(0, T ; W)} \leq c .
$$

Therefore, by weak compactness, there exists some $z$ such that

$$
z_{n} \rightarrow z \quad \text { weakly star in } H^{1}(0, T ; H) \cap L^{\infty}(0, T ; V) \cap L^{2}(0, T ; W),
$$

at least for a subsequence. This also implies $z_{n} \rightarrow z$ weakly in $C^{0}([0, T]$; $H)$ and, recalling (37), we infer that

$$
a \partial_{t} z+\partial_{t} a z-\Delta z=b \quad \text { and } \quad z(0)=\mu_{0} .
$$

Now, we apply Corollary 3.2 and conclude that $z=\mu$, whence (36) follows. The last assertion is a consequence of the well-known embedding $H^{1}(0, T ; H) \cap L^{2}(0, T ; W) \subset C^{0}([0, T] ; V)$.

The regularity just established can be improved provided that a stronger assumption on the initial datum $\mu_{0}$ is satisfied, namely

$$
\mu_{0} \in W
$$

We observe that the regularity given in (42) implies (13) because of the continuous embedding $W \subset C^{0}(\bar{\Omega})$. The new regularity result is stated in the following theorem, whose proof is performed with the same technique as before.

THEOREM 3.4. In addition to (9)-(14), assume that (42) bolds, and let $(\mu, \rho)$ be a solution to (18)-(20) satisfying (15)-(17). Then $\mu$ also satisfies

$$
\partial_{t} \mu \in L^{\infty}(0, T ; H) \cap L^{2}(0, T ; V) \text { and } \mu \in L^{\infty}(0, T ; W) .
$$

PROOF. Theorem 3.3 ensures that (36) holds for $\mu$, so that the regularity of $\partial_{t} \rho, a$, and $b$ can be updated. Indeed, (36) implies that (19) can 
be differentiated with respect to time and that $\partial_{t}^{2} \rho \in L^{2}(Q)$. Hence, we also have

$$
\partial_{t}^{2} a \in L^{2}(Q) \quad \text { and } \quad \partial_{t} b \in L^{2}(Q),
$$

which allows us to construct a new approximation of problem (35). Namely, we can choose sequences $\left\{a_{n}\right\}$ and $\left\{b_{n}\right\}$ of $C^{2}$ functions satisfying (37)-(38) and

$$
\left\|\partial_{t}^{2} a_{n}\right\|_{L^{2}(Q)}+\left\|\partial_{t} b_{n}\right\|_{L^{2}(Q)} \leq c \quad \text { for every } n .
$$

This leads to a sequence of solutions $z_{n}$ to the corresponding problems (39), which, however, still keeps all of the properties of the approximation we had established in the proof of the previous theorem. But the regularity of the coefficient and the assumption (42) on $\mu_{0}$ also ensure us that $z_{n}$ is smoother and that equation (39) can be differentiated with respect to time. By doing this, we get

$$
a_{n} \partial_{t}^{2} z_{n}+2 \partial_{t} a_{n} \partial_{t} z_{n}+\partial_{t}^{2} a_{n} z_{n}-\Delta \partial_{t} z_{n}=\partial_{t} b_{n},
$$

and we can multiply this equality by $\partial_{t} z_{n}$ and integrate over $Q_{t}$, where $t \in(0, T)$ is arbitrary. By integrating by parts with respect to time the term involving the second derivative $\partial_{t}^{2} z_{n}$, and owing to the inequality $a_{n} \geq 1$, we obtain

$$
\begin{aligned}
\frac{1}{2} \int_{\Omega}\left|\partial_{t} z_{n}(t)\right|^{2}+\int_{Q_{t}}\left|\nabla \partial_{t} z_{n}\right|^{2} & \\
\leq & \frac{1}{2} \int_{\Omega} a_{n}(0)\left|\partial_{t} z_{n}(0)\right|^{2}-\frac{5}{2} \int_{Q_{t}} \partial_{t} a_{n}\left|\partial_{t} z_{n}\right|^{2} \\
& -\int_{Q_{t}} \partial_{t}^{2} a_{n} z_{n} \partial_{t} z_{n}+\int_{Q_{t}} \partial_{t} b_{n} \partial_{t} z_{n} .
\end{aligned}
$$

Two of the terms on the right-hand side can be dealt with, by accounting for (38), (41) and (45), in the following way: 


$$
-\frac{5}{2} \int_{Q_{t}} \partial_{t} a_{n}\left|\partial_{t} z_{n}\right|^{2}+\int_{Q_{t}} \partial_{t} b_{n} \partial_{t} z_{n} \leq c+c \int_{Q_{t}}\left|\partial_{t} z_{n}\right|^{2} \leq c .
$$

For the first one, we observe that (39), (42), and (38) imply that $a_{n}(0) \leq c$ and

$$
\begin{aligned}
\left|\partial_{t} z_{n}(0)\right| & \leq\left|b_{n}(0)+\Delta z_{n}(0)-\partial_{t} a_{n}(0) z_{n}(0)\right| \\
& \leq c+\left|\Delta \mu_{0}\right|+c \mu_{0} \leq c+\left|\Delta \mu_{0}\right| .
\end{aligned}
$$

As $\mu_{0} \in W$, the integral under investigation is bounded. It remains to handle the third term. By using first the Hölder and Young inequalities, and then the Sobolev and compactness inequalities (23) and (24) with $q=4$, we have

$$
\begin{aligned}
-\int_{Q_{t}} \partial_{t}^{2} a_{n} z_{n} \partial_{t} z_{n} & \leq \int_{0}^{t}\left\|\partial_{t}^{2} a_{n}(s)\right\|_{2}\left\|z_{n}(s)\right\|_{4}\left\|\partial_{t} z_{n}(s)\right\|_{4} d s \\
& \leq \int_{0}^{t}\left\|\partial_{t} z_{n}(s)\right\|_{4}^{2}+\int_{0}^{t}\left\|\partial_{t}^{2} a_{n}(s)\right\|_{2}^{2}\left\|z_{n}(s)\right\|_{4}^{2} d s
\end{aligned}
$$

whence

$$
\begin{aligned}
-\int_{Q_{t}} \partial_{t}^{2} a_{n} z_{n} \partial_{t} z_{n} \leq & \frac{1}{2} \int_{0}^{t}\left\|\nabla \partial_{t} z_{n}(s)\right\|_{H}^{2} d s+c \int_{0}^{t}\left\|\partial_{t} z_{n}(s)\right\|_{H}^{2} d s \\
& +c \int_{0}^{t}\left\|\partial_{t}^{2} a_{n}(s)\right\|_{2}^{2}\left\|z_{n}(s)\right\|_{V}^{2} d s \\
\leq & \frac{1}{2} \int_{0}^{t}\left\|\nabla \partial_{t} z_{n}(s)\right\|_{H}^{2} d s+c,
\end{aligned}
$$

where the last inequality follows from (41) and (45). By collecting all this and (46) and rearranging, we conclude that

$$
\left\|\partial_{t} z_{n}\right\|_{L^{\infty}(0, T ; H)}+\left\|\partial_{t} z_{n}\right\|_{L^{2}(0, T ; V)} \leq c
$$


Moreover, by comparison in (39), we also infer that $\left\{\Delta z_{n}\right\}$ is bounded in $L^{\infty}(0, T ; H)$. Elliptic regularity and (41) then yield the boundedness of $\left\{z_{n}\right\}$ in $L^{\infty}(0, T ; W)$. At this point, we use weak compactness once more. We obtain, on a subsequence, that

$z_{n} \rightarrow z \quad$ weakly star in $W^{1, \infty}(0, T ; H) \cap H^{1}(0, T ; V) \cap L^{\infty}(0, T ; W)$

and conclude that $z=\mu$ as before. Hence, (36) holds, and the proof is complete.

COROLLARY 3.5. Under the assumptions of Theorem 3.4, every solution $(\mu, \rho)$ to problem (18)-(20) also satisfies

$$
\partial_{t} \mu \in L^{10 / 3}(Q) .
$$

PROOF. It suffices to combine the first of (43) and (25).

The regularity just achieved allows us to give a rather simple uniqueness proof.

THEOREM 3.6. Assume that (9)-(14) and (42) bold. Then the solution $(\mu, \rho)$ given by Theorem 2.1 is unique.

PROOF. We adapt the argument used in [7] to the present situation. We pick two solutions $\left(\mu_{i}, \rho_{i}\right), i=1,2$, and set for convenience

$$
\begin{aligned}
\rho & :=\rho_{1}-\rho_{2}, \\
\mu & :=\mu_{1}-\mu_{2}, \\
\gamma_{i} & :=g\left(\rho_{i}\right), \\
\gamma & :=\gamma_{1}-\gamma_{2}, \\
\eta_{i} & :=g^{\prime}\left(\rho_{i}\right), \\
\eta & :=\eta_{1}-\eta_{2} .
\end{aligned}
$$

By accounting for Remark 2.2, we write (21) for both solutions, take the difference, and multiply by $\mu$. At the same time, we write (19) for both solutions, add $\rho_{i}$ to both sides for convenience, move all the nonlinear 
terms to the right-hand side, and multiply the difference by $\partial_{t} \rho$. Then, we integrate and sum up. By owing to the identity

$$
\begin{aligned}
& \left\{\left(1+2 \gamma_{1}\right) \partial_{t} \mu_{1}+\mu_{1} \eta_{1} \partial_{t} \rho_{1}-\left(1+2 \gamma_{2}\right) \partial_{t} \mu_{2}+\mu_{2} \eta_{2} \partial_{t} \rho_{2}\right\} \mu \\
& \quad=\frac{1}{2} \partial_{t}\left\{\left(1+2 \gamma_{1}\right)|\mu|^{2}\right\}+2 \gamma \partial_{t} \mu_{2} \mu+\mu_{2} \eta \partial_{t} \rho_{1} \mu+\mu_{2} \eta_{2} \partial_{t} \rho \mu,
\end{aligned}
$$

and using the boundedness and the Lipschitz continuity of the nonlinearities, we obtain

$$
\begin{aligned}
& \frac{1}{2} \int_{\Omega}|\mu(t)|^{2}+\int_{Q_{t}}|\nabla \mu|^{2}+\int_{Q_{t}}\left|\partial_{t} \rho\right|^{2}+\frac{1}{2} \int_{\Omega}|\rho(t)|^{2} \\
& \quad \leq c \int_{Q_{t}}\left|\partial_{t} \mu_{2}\right||\rho||\mu|+c \int_{Q_{t}}\left(|\rho||\mu|+\left|\partial_{t} \rho\right||\mu|+\left|\partial_{t} \rho\right||\rho|\right) .
\end{aligned}
$$

Just the first integral on the right-hand side needs some treatement. By the Hölder inequality, the compactness inequality (24) with $q=5$, and the elementary Young inequality, we have for any $\varepsilon>0$

$$
\begin{aligned}
\int_{Q_{t}}\left|\partial_{t} \mu_{2}\right||\rho||\mu| \leq & \int_{0}^{t}\left\|\partial_{t} \mu_{2}(s)\right\|_{10 / 3}\|\rho(s)\|_{2}\|\mu(s)\|_{5} d s \\
\leq & \int_{0}^{t}\|\mu(s)\|_{5}^{2} d s+\int_{0}^{t}\left\|\partial_{t} \mu_{2}(s)\right\|_{10 / 3}^{2}\|\rho(s)\|_{2}^{2} d s \\
\leq & \varepsilon \int_{0}^{t}\|\nabla \mu(s)\|_{2}^{2} d s+c_{\varepsilon} \int_{0}^{t}\|\mu(s)\|_{2}^{2} d s \\
& +\int_{0}^{t}\left\|\partial_{t} \mu_{2}(s)\right\|_{10 / 3}^{2}\|\rho(s)\|_{2}^{2} d s .
\end{aligned}
$$

As the function $s \mapsto\left\|\partial_{t} \mu_{2}(s)\right\|_{10 / 3}^{2}$ belongs to $L^{5 / 3}(0, T)$ by Corollary 3.5, and thus to $L^{1}(0, T)$, the last integral can be controlled by the left-hand side of (48) via Gronwall-Bellman's lemma (see, e.g., [14, 
Lemma A.4, p. 156]). Hence, it is sufficient to choose $\varepsilon$ small enough and apply this lemma in order to conclude that $\mu=0$ and $\rho=0$.

Remark 3.7. It is clear that the bootstrap procedure used in the above proofs can be continued to provide even more regularity for the solution $(\mu, \rho)$ to problem (18)-(20) under suitable assumptions on the initial data.

\section{ACKNOWLEDGMENTS}

Pierluigi Colli and Gianni Gilardi gratefully acknowledge some financial support form the MIUR-PRIN Grant 2010A2TFX2 "Calculus of Variations" and the GNAMPA (Gruppo Nazionale per l'Analisi Matematica, la Probabilità e le loro Applicazioni) of INdAM (Istituto Nazionale di Alta Matematica).

\section{REFERENCES}

[1] P. Colli, G. Gilardi, P. Krejčí, J. Sprekels, A vanishing diffusion limit in a nonstandard system of phase field equations, Evol. Equ. Control Theory, 2014: 3: 257-275.

[2] P. Colli, G. Gilardi, P. Krejčí, J. Sprekels, A continuous dependence result for a nonstandard system of phase field equations, Math. Methods Appl. Sci., 2014: 37: 1318-1324.

[3] P. Podio-Guidugli, Models of phase segregation and diffusion of atomic species on a lattice, Ric. Mat., 2006: 55: 105-118.

[4] E. Fried and M.E. Gurtin, Continuum theory of thermally induced phase transitions based on an order parameter, Phys. D, 1993: 68: 326-343.

[5] M.E. Gurtin, Generalized Ginzburg-Landau and Cahn-Hilliard equations based on a microforce balance, Phys. D, 1996: 92: 178192. 
[6] P. Colli, G. Gilardi, P. Krejčí, P. Podio-Guidugli, J. Sprekels, Analysis of a time discretization scheme for a nonstandard viscous CahnHilliard system, ESAIM Math. Model. Numer. Anal., 2014: 48: 1061-1087.

[7] P. Colli, G. Gilardi, P. Podio-Guidugli, J. Sprekels, Well-posedness and long-time behaviour for a nonstandard viscous Cahn-Hilliard system, SIAM J. Appl. Math., 2011: 71: 1849-1870.

[8] P. Colli, G. Gilardi, P. Podio-Guidugli, J. Sprekels, An asymptotic analysis for a nonstandard Cahn-Hilliard system with viscosity, Discrete Contin. Dyn. Syst. Ser. S, 2013: 6: 353-368.

[9] P. Colli, G. Gilardi, P. Podio-Guidugli, J. Sprekels, Distributed optimal control of a nonstandard system of phase field equations, Contin. Mech. Thermodyn., 2012: 24: 437-459.

[10] P. Colli, G. Gilardi, P. Podio-Guidugli, J. Sprekels, Global existence and uniqueness for a singular/degenerate Cahn-Hilliard system with viscosity, J. Differential Equations, 2013: 254: 4217-4244.

[11] P. Colli, G. Gilardi, J. Sprekels, Analysis and optimal boundary control of a nonstandard system of phase field equations, Milan J. Math., 2012: 80: 119-149.

[12] P. Colli, G. Gilardi, P. Podio-Guidugli, J. Sprekels, Global existence for a strongly coupled Cahn-Hilliard system with viscosity, Boll. Unione Mat. Ital. (9), 2012: 5: 495-513.

[13] P. Colli, G. Gilardi, P. Podio-Guidugli, J. Sprekels, Continuous dependence for a nonstandard Cahn-Hilliard system with nonlinear atom mobility, Rend. Semin. Mat. Univ. Politec. Torino, 2012: 70: 27-52.

[14] H. Brezis, Opérateurs maximaux monotones et semi-groupes de contractions dans les espaces de Hilbert, North-Holland Math. Stud. 5, North-Holland, Amsterdam, 1973. 